\title{
Supporting Information for: Molecular Dynamics Simulations of Hydration Effects on Solvation, Diffusivity, and Permeability in Chitosan/Chitin Films
}

By Marshall T. McDonnell ${ }^{\dagger}$ Duncan Greeley ${ }^{*}$ Kevin Kit , and David J. Keffert,*

${ }^{\dagger}$ Department of Chemical and Biomolecular Engineering, and Department of Materials

Science and Engineering, University of Tennessee, Knoxville, TN 37996, United States

*Corresponding Author: dkeffer@utk.edu 


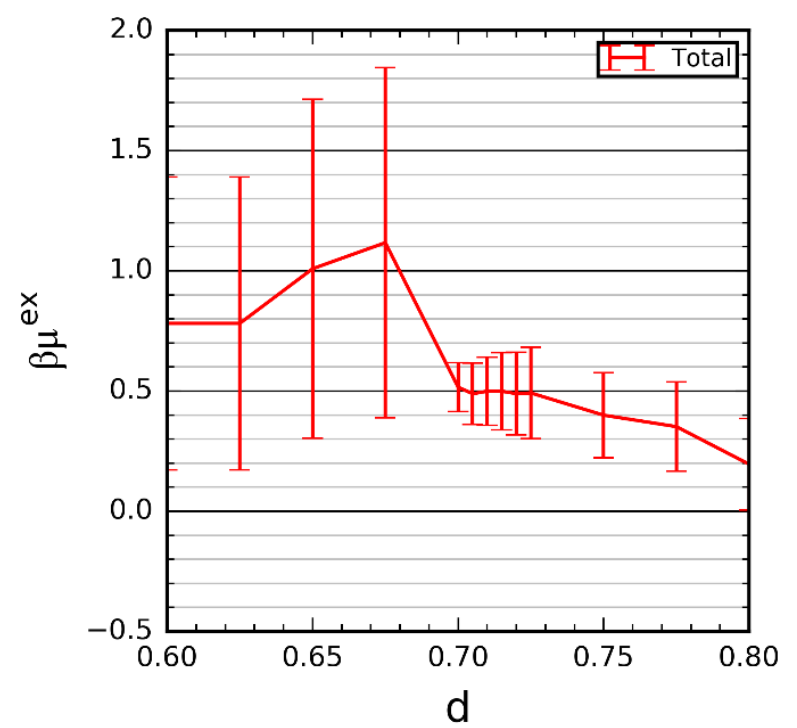

Figure S1. Reduce chemical potential vs. hard-core diameter for oxygen for $15 \% \mathrm{RH}$ chitosan/chitin membrane system. Best choice of diameter is chosen in the plateau-region and where the smallest standard error is given (here $d=0.700$ ).

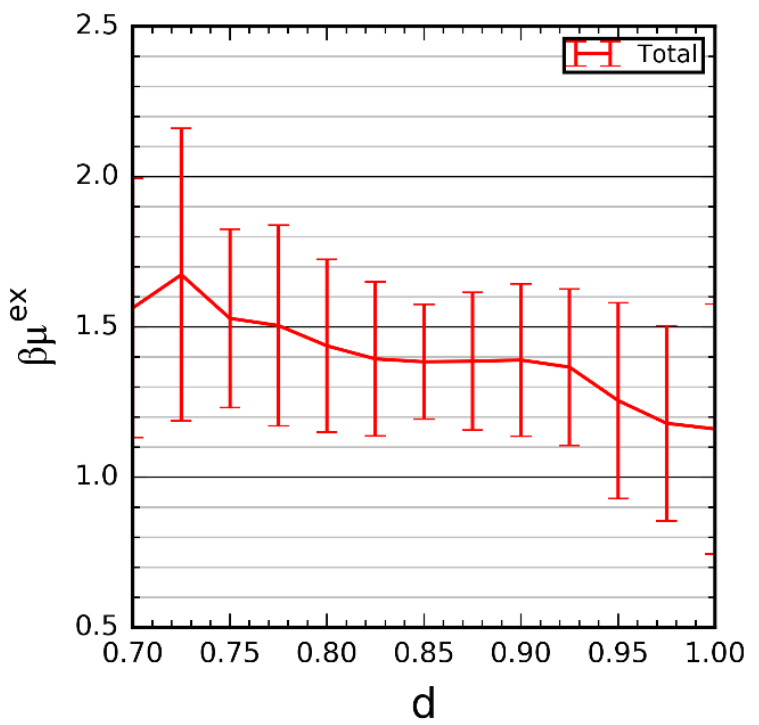

Figure S2. Reduce chemical potential vs. hard-core diameter for oxygen for 95\% RH chitosan/chitin membrane system. Best choice of diameter is chosen in the plateau-region and where the smallest standard error is given (here $d=0.850$ ). 